\title{
Identification of Quantitative Trait Loci for Resistance Against Verticillium longisporum in Oilseed Rape (Brassica napus)
}

\author{
W. Rygulla, R. J. Snowdon, W. Friedt, I. Happstadius, W. Y. Cheung, and D. Chen
}

First, second, and third authors: Department of Plant Breeding, Research Centre for BioSystems, Land Use and Nutrition, Justus Liebig University, Heinrich-Buff-Ring 26-32, D-35392 Giessen, Germany; fourth author: Svalöf Weibull AB, S-268 81 Svalöv, Sweden; and fifth and sixth authors: DNA Landmarks Inc., Saint-Jean-sur-Richelieu 84, Quebec, Canada.

Accepted for publication 19 September 2007.

\begin{abstract}
Rygulla, W., Showdon, R. J., Friedt, W., Happstadius, I., Cheung, W. Y., and Chen, D. 2008. Identification of quantitative trait loci for resistance against Verticillium longisporum in oilseed rape (Brassica napus). Phytopathology 98:215-221.

Verticillium longisporum is one of the major pathogens of oilseed rape (Brassica napus; genome AACC, $2 \mathrm{n}=38$ ) in Europe. Current European cultivars possess only a low level of resistance against $V$. longisporum, meaning that heavy infection can cause major yield losses. The aim of this study was to identify quantitative trait loci (QTL) for resistance against $V$. longisporum as a starting point for marker-assisted breeding of resistant cultivars. Resistance QTL were localized in a segregating oilseed rape population of 163 doubled haploid $(\mathrm{DH})$ lines derived by microspore culture from the $\mathrm{F}_{1}$ of a cross between two B. napus breeding lines, one of which exhibited $V$. longisporum resistance derived by pedigree selection from a resynthesized B. napus genotype. A genetic map was constructed comprising 165 restriction fragment length polymorphism, 94 amplified fragment length polymorphism and 45 simple sequence repeats (SSR)

markers covering a total of $1,739 \mathrm{cM}$ on 19 linkage groups. Seedlings of the DH lines and parents were inoculated with $V$. longisporum isolates in four greenhouse experiments performed in Sweden during autumn 1999. In three of the experiments the DH lines were inoculated with a mixture of five isolates, while in the fourth experiment only one of the isolates was used. The intention was to simulate four different environments with variable disease pressure, while still maintaining uniform conditions in each environment to enable reliable disease scoring. The disease index (DI) was calculated by scoring symptoms on a total of 21 inoculated plants per line in comparison to 21 noninoculated plants per line. Using the composite interval mapping procedure a total of four different chromosome regions could be identified that showed significant QTL for resistance in more than one environment. Two major QTL regions were identified on the C-genome linkage groups N14 and N15, respectively; each of these QTL consistently exhibited significant effects on resistance in multiple environments. The presence of flanking markers for the respective QTL was associated with a significant reduction in DI in the inoculated DH lines.
\end{abstract}

Verticillium wilt caused by Verticillium longisporum (ex. Verticillium dahliae var. longisporum Stark) is one of the major diseases of oilseed rape $(2 \mathrm{n}=38$, genome AACC) in Europe. Current European cultivars possess only a low level of resistance against $V$. longisporum, meaning that heavy infection can cause major yield losses $(9,34)$. Because long-term crop rotation is not always possible and no efficient chemical control is available, the disease is becoming a serious problem in some areas of Germany, France, Sweden, and other major European rapeseed-producing countries (14). The fungus infects the plant via the roots by penetrating the root epidermis in the root hair zone, and establishes itself in the xylem $(10,35)$. Once it has reached the xylem vessels it forms conidiospores, which spread inside the xylem throughout the vegetative parts of the plant. Symptoms usually appear late in the growing season on mature plants, when diseased plants wilt and often die. The fungus produces microsclerotia in plant debris that can survive in the soil for long periods of time.

Brassica napus is a young species that originated through spontaneous interspecific hybridizations between B. rapa L. (syn. campestris; genome AA, $2 \mathrm{n}=20)$ and B. oleracea $\mathrm{L}$. $(\mathrm{CC}, 2 \mathrm{n}=$ 18) (26). After intensive breeding for oil and seed quality traits, the spring and winter oilseed forms of B. napus now represent one of the most important sources of vegetable oil worldwide. Due to an extremely strong bottleneck selection for zero seed erucic acid

Corresponding author: R. J. Snowdon

E-mail address: Rod.Snowdon@agrar.uni-giessen.de

doi:10.1094/PHYTO-98-2-0215

(C) 2008 The American Phytopathological Society and low seed glucosinolate content, current oilseed rape cultivars have a comparably narrow genetic basis (12); in particular, the availability of novel germplasm for resistance breeding is often extremely limited. On the other hand, sources of Verticillium resistance that can potentially be used in rapeseed breeding have been identified in B. oleracea and B. rapa $(6,11,24,25)$. For example, Happstadius et al. (11) selected the most resistant $B$. oleracea accessions identified in a greenhouse screening and demonstrated that their resistance was effective against $V$. longisporum when introduced into resynthesized (RS) B. napus breeding lines by interspecific hybridization. We have produced further RS rapeseed breeding lines with high levels of resistance from cabbage accessions combined with oilseed turnip rape $(24,25)$, while Debode et al. (6) identified resistance in cauliflower cultivars and were able to show that resistant cultivars can prevent the pathogen from spreading systemically inside the plant. The genetic basis of these resistance sources is not known, however, and molecular markers linked to resistance loci and therefore exploitable for marker-assisted selection have not previously been described for this disease. The aim of this study was to identify quantitative trait loci (QTL) for resistance against $V$. longisporum in order to gain information on the genetics of new, resistant $B$. napus breeding lines. The study represents a first step towards marker-assisted breeding of resistant oilseed rape cultivars and, in the long term, towards the map-based cloning of the responsible resistance genes.

\section{MATERIALS AND METHODS}

Resistance donor and mapping population. The original resistance donor for the mapping population used in the QTL 
analysis was a tetraploid white cabbage (B. oleracea convar. capitata; genome CCCC, $2 \mathrm{n}=4 \times=36$ ), which was crossed with a tetraploid, low erucic acid winter turnip rapeseed (B. rapa ssp. olerifera; genome AAAA, $2 \mathrm{n}=4 \times=40$ ) to generate a synthetic B. napus. This breeding line was produced in Sweden in 1975 to 1976 with the aim of increasing the genetic variability in B. napus germplasm, and the resistance was subsequently transferred to oilseed rape breeding lines by pedigree selection. The parents of the mapping population were homozygous doubled haploid (DH) breeding lines that were identified by greenhouse screenings of two $\mathrm{DH}$ populations for resistance to $V$. longisporum. The parental line 307-406-1 was found to be susceptible to Verticillium wilt, while the parental line 307-230-2 was considered to be resistant/tolerant to the disease. Three $\mathrm{F}_{1}$ donor plants were used for microspore cultivation as described in Zhou et al. (36). A total of 3,453 in vitro embryos were induced, 478 shoots were regenerated, and 398 plants were transplanted to soil. At the three- to four-leaf stage the ploidy number of each plant was analyzed by flow cytometry using standard methods (33). In $40 \%$ of the plantlets, the genome had doubled spontaneously, $6 \%$ were polyploids, and $54 \%$ were still haploid and consequently treated with colchicine to induce diploidization. After vernalization, 329 plants were transferred to the greenhouse and selfed by bagging. One hundred and sixty-three randomly chosen DH lines were used for the QTL mapping study.

Resistance screening. Seedlings of the DH lines and parents were inoculated with $V$. longisporum isolates in four separate greenhouse experiments during autumn 1999 at Svalöf Weibull $\mathrm{AB}$, Sweden. The intention was to simulate four different environments with variable disease pressure, while still maintaining uniform conditions in each environment to enable reliable disease scoring. Since the resistance was expected to be quantitative in nature, this strategy was used to enable more reliable identification of QTL that were stable over different environments.

In three of the experiments a mixture of the Swedish V. longisporum isolates $\mathrm{Vd} 1, \mathrm{Vd} 4, \mathrm{Vd} 11, \mathrm{Vd} 12$, and $\mathrm{Vd} 13$ in equal densities was used for the inoculation. The characteristics of these five isolates, which show a similar virulence spectrum and moderate to high aggressiveness, have been described in detail previously $(27,28)$. In the fourth experiment, the inoculation was performed with the single-spore isolate $\mathrm{Vd13}$, in order to determine if any identified QTL might be explained by a racespecific resistance. Vd13 shows consistent, moderate disease symptoms, disease severity, and DI with different inoculation techniques in susceptible $B$. napus cultivars (28). The same overall inoculum density $\left(2 \times 10^{6}\right.$ spores $/ \mathrm{ml}+$ mycelium fragments $)$ was used for the inoculations with the isolate mixture and for the single-spore isolate.

Each experiment included a total of 21 inoculated and 21 noninoculated plants for each of the DH lines, along with 10 repetitions of 21 inoculated and 21 noninoculated plants of each of the parental lines. Furthermore, 21 inoculated and 21 noninoculated plants of the susceptible B. napus 'Libraska' were included in each experiment as an infection control, making a total of 3,654 inoculated and 3,654 noninoculated plants in each of the four experiments. The plants were grown in individual pots with the genotypes arranged in a completely random design. The inoculations were carried out using a root-dip inoculation method that was shown in a comparison of different Verticillium inoculation techniques to give the most consistent results (15): Bottombroken peat pots containing 14-day-old seedlings were dipped into $40 \mathrm{ml}$ of inoculum. The pots were left in the inoculum suspension for $30 \mathrm{~min}$ and then replaced at the original position in a planting tray. The remaining inoculum was recovered with rinsing water and poured into the container with the inoculated seedlings. The seedlings were then grown at $20^{\circ} \mathrm{C}$ in a greenhouse with a light regime of $16 \mathrm{~h}$ day $/ 8 \mathrm{~h}$ night; the relative humidity was not controlled in order to allow environmental variation between the four experiments in the interests of the QTL analysis. Disease scoring was carried out between 8 to 10 weeks after inoculation, when 80 to $90 \%$ of the inoculated plants from $B$. napus Libraska showed wilting symptoms.

Disease severity scores (0 to 5) for each inoculated plant followed a scale (28) based on the percentage of leaves showing wilting and chlorosis, along with the degree of stunted growth (height in $\mathrm{mm}$ ) in comparison to the mean of the noninoculated plants of the same genotype: $0=$ no symptoms, $1=$ wilting and/or stunting $<25 \%, 2=$ wilting and/or stunting 25 to $50 \%, 3=$ wilting and/or stunting 51 to $90 \%, 4=$ wilting and stunting $\geq 91 \%$, and $5=$ dead plant. From the number of plants in each class, DI was calculated as follows for each of the DH lines and parents, as described by Happstadius et al. (11):

$$
\begin{aligned}
& \mathrm{DI}=\frac{\left(0 \times N_{0}\right)+\left(1 \times N_{1}\right)+\left(2 \times N_{2}\right)+\left(3 \times N_{3}\right)+\left(4 \times N_{4}\right)+\left(5 \times N_{5}\right)}{\left(N_{0}+N_{1}+N_{2}+N_{3}+N_{4}+N_{5}\right)}- \\
& \left(\mathrm{DI}_{\mathrm{n}, \text { control }}-\mathrm{DI}_{\text {total, control }}\right)
\end{aligned}
$$

where $N_{n}$ is the number of plants in the respective class, $D_{n}$, control represents the mean symptom score of the noninoculated control plants in the $n$th trial, and $\mathrm{DI}_{\text {total, control }}$ is the mean symptom score of the noninoculated controls over all trials.

Statistical analyses of the data were performed using the software package SAS 9.1. Analysis of variance (ANOVA) was performed using the general linear model procedure in order to estimate differences within the population and among the four different experiments (environments). ANOVA was also used to analyze genotype-environment interactions of the two parental lines, for which 10 DI values (each calculated from 21 inoculated and 21 noninoculated plants) per line per experiment were available. Variation in DI within and among the four experiments was further quantified by calculation of least significant differences among the means of the parents and the DH lines, along with Pearson's correlation coefficients for the individual genotypes in the four different environments.

Restriction fragment length polymorphism (RFLP) probes and Southern analysis. Plant genomic DNA was extracted for RFLP analysis as described by Cheung et al. (4). Three restriction enzymes (EcoRI, EcoRV, and HindIII) were applied to digest the genomic DNA. The DNA digests were separated in $0.8 \%$ agarose gels and transferred to nylon membranes (H-bond $\mathrm{N}+$ ). Six sets of DNA membranes for each enzyme digest were prepared for Southern analysis. DNA probes coded DLM2 to DLM372 correspond to anonymous clones from a seedling cDNA library of $B$. napus. Procedures for Southern analysis were described by Cheung et al. (4). Duplicated loci detected by a single clone were designated by the same name followed by a different lowercase letter, as described by Landry et al. (17).

Amplified fragment length polymorphism (AFLP) and simple sequence repeats (SSR) analysis. Marker saturation of the genetic map was achieved using AFLP markers, while previously mapped loci from publicly-available SSR primer combinations were also included in the map to enable identification of linkage groups. For the polymerase chain reaction (PCR) marker analyses new genomic DNA samples were isolated from dry frozen leaf material according to Doyle and Doyle (8). AFLP amplification products were generated using the EcoRI-MseI-based AFLP kit of Invitrogen (AFLP Core Reagent Kit, Invitrogen Life Technologies Inc., Carlsbad, CA) according to the manufacturer's instructions. EcoRI primers were fluorescently labeled with IRD700 or IRD800. DNA from DH lines and their parents were amplified with a set of 10 AFLP primer combinations known either from the literature (18) or from previous mapping studies $(1,2)$ to reveal a high polymorphism rate in oilseed rape.

A total of 127 SSR primer combinations for which genetic map positions of one or more loci in B. napus are known $(2,19,23)$, were screened for polymorphism among the mapping parents. Of 
these primer combinations $65(51.2 \%)$ amplified at least one polymorphic locus and were screened in the DH lines. For all SSR analyses the M13-tailing procedure described by Berg and Olaisen (3) was used. In this method the fluorescently labeled universal M13 primer 5'-AGGGTTTTCCCAGTCACGACGTT-3' is added to the PCR reaction, and the forward primer of each SSR is appended with the sequence 5'-TTTCCCAGTCACGACGTT$3^{\prime}$. After the first cycle of amplification the PCR fragments are subsequently amplified by the labelled universal primer. All AFLP and SSR amplification products were separated using a LI-COR 4200 DNA Analyzer (LI-COR Biosciences, Lincoln, NE) and scored visually.

Segregation analysis and map construction. A genetic map was constructed using the program JoinMap 3.0 (31). The fit of allelic segregation to the expected 1:1 segregation ratio was tested for each marker by a chi-square test, and map construction was performed in three steps based on the suggestions of Cloutier et al. (5) for B. napus mapping. (i) Selection of loci whose segregation fit the expected ratio $(P<0.01)$ to identify linkage groups with high confidence; these markers were anchored as "fixed orders". (ii) Addition of markers with segregation other than the expected 1:1 ratio $(P \geq 0.01)$ using a minimum logarithm of odds (LOD) threshold of 2.0. These markers were only added when they did not influence the grouping of the fixed-order markers and did not form noticeable clusters with other skewed markers. (iii) Identification of linkage groups based on SSR markers from consensus maps $(1,2,23)$. Map distances measured in cM between markers were derived from the Kosambi function (16).

Composite interval mapping of resistance QTL. QTL conferring resistance to $V$. longisporum in the $\mathrm{DH}$ lines were localized by composite interval mapping with cofactors, using the computer software PLABQTL (30) Version 1.2. All markers proposed by the default option SELECT were used as cofactors. Phenotype data from each of the four experiments were first analyzed separately, then the data from the three experiments with the isolate mixture were averaged to compare the locations of QTL detected over different environments under the same infection conditions. Initially, all putative QTL for resistance were identified using an LOD threshold of 3.0. From these putative QTL, the ones exhibiting a significant likelihood ratio that a QTL is present versus absent were identified based on the multiple regression of the final simultaneous fit function of PLABQTL. After a further regression analysis based on the phenotypic variance, only QTL that showed a significant LOD score in combination with a significant phenotypic effect $(\theta=0.02)$ were selected, and we considered these to be trait-relevant QTL only when the confidence intervals overlapped in two or more of the four experiments.

\section{RESULTS AND DISCUSSION}

Phenotypic evaluation of the mapping population. Figure 1 shows the distribution of DI values for the DH lines in the four experiments. In all cases, a continuous distribution was observed, with the majority of DH lines having an intermediate DI between the two parents; this indicates a quantitative resistance for both the isolate mixture and the single-spore isolate. For both the single-spore isolate and the isolate mixture, a number of DH lines showed a lower DI than the resistant parent, or a higher susceptibility than the susceptible parent, suggesting a transgressive segregation with some resistance alleles also being contributed by the susceptible parent. In the experiment with the single-spore isolate, the DI values in the DH population showed a more or less normal distribution, with the majority of the DH lines showing an intermediate resistance phenotype and few lines being highly susceptible or highly resistant. On the other hand, in the three experiments with the isolate mixture, both the distribution and the mean DI values of the two parents were strongly skewed towards greater susceptibility. This indicates that the isolate mixture raises the disease pressure and may overcome part of the resistance against the single-spore isolate.

Considerable variation was sometimes observed among the symptom scores of individual plants within the same DH line,
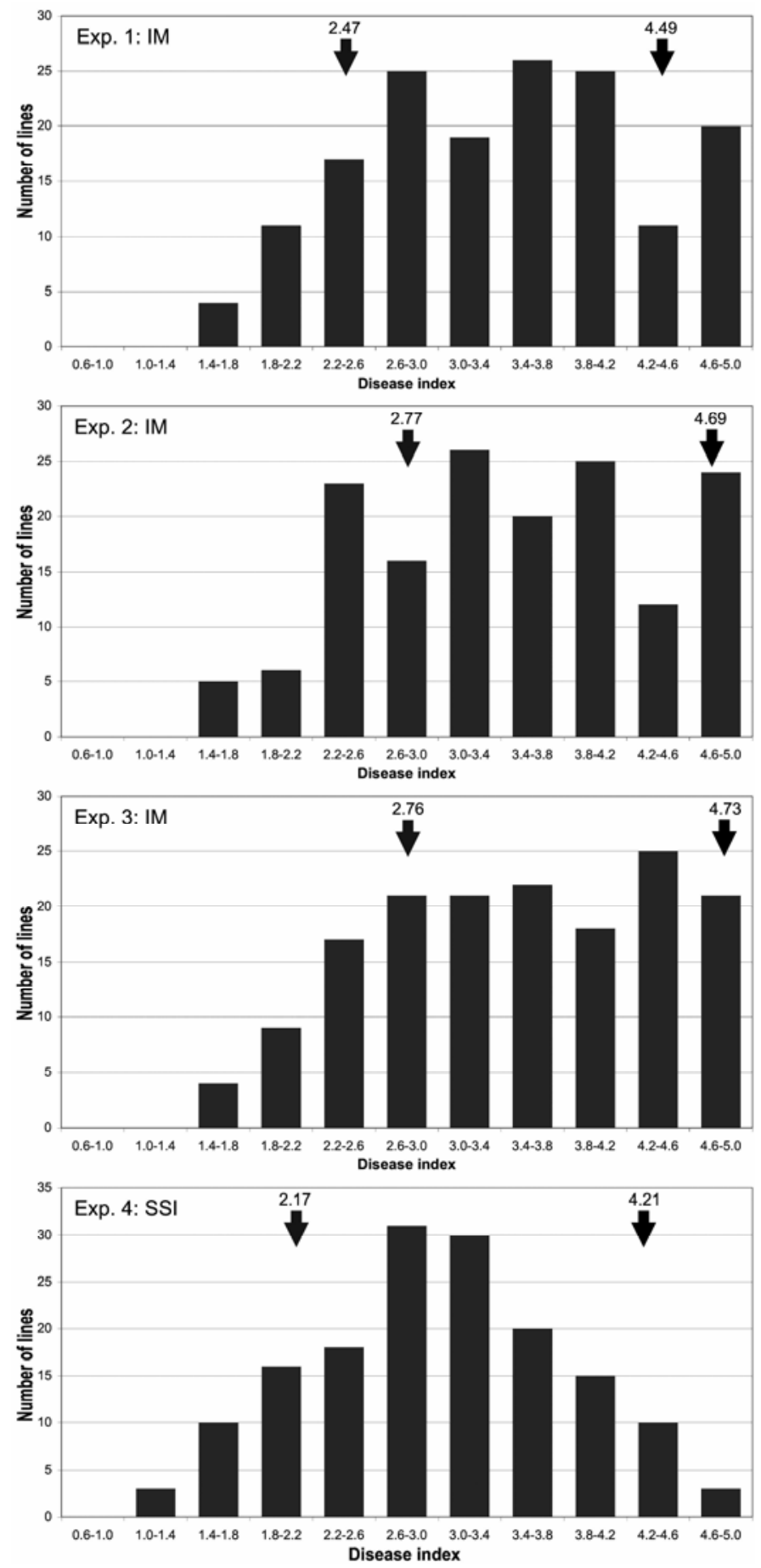

Fig. 1. Distributions of Verticillium longisporum disease index (DI) values in a population of 163 doubled-haploid Brassica napus lines derived from a single $\mathrm{F}_{1}$ individual from the population 307-230-2 (resistant)/307-406-1 (susceptible). The disease index was calculated from symptom scores on 21 plants per line after root-dip inoculation, compared to 21 noninoculated plants per line, in four greenhouse experiments in autumn 1999. In experiments 1 to 3, an isolate mixture was used, while experiment 4 was performed by inoculation with a single spore isolate. The left and right arrows show the mean DI values of the resistant and susceptible parents, respectively; parental means were calculated from 10 repetitions of 21 inoculated and 21 noninoculated plants in each of the four experiments. Exp.: experiment; IM: isolate mixture; SSI: single-spore isolate. 
possibly indicating that variable amounts of inoculum may be taken up during the disease screening. Despite this variation at a single-plant level, statistical analysis of the DI data showed that a reliable and reproducible quantitative estimate of the disease score was still obtained by calculating the DI from 21 inoculated plants, compared to 21 noninoculated plants in each experiment. Since the mean DI values of the parents and the DH lines showed high reproducibility over the three different experiments with the isolate mixture, we decided to also include the DI values from the inoculation with the single-spore isolate in the QTL analysis, even though only one experimental repetition was available in this case. In the ANOVA, the DI values of the parents and the DH lines were significantly lower $(P<0.01)$ after inoculation with the single-spore isolate than after the inoculations with the isolate mixture; this difference can be clearly seen in the distributions shown in Figure 1 and was confirmed by the results of the least significant difference (LSD) test (Table 1). Despite this difference in overall symptom level, however, the mean DI values of the individual genotypes infected with the single-spore isolate were still significantly correlated $(P<0.01)$ to the mean DI values after the inoculations with the isolate mixture (Table 2). This is presumably because a large number of DH lines were resistant against both the single-spore isolate and the isolate mixture. The ANOVA also revealed that there was no significant genotype $x$ environment interaction $(P=0.96)$ over the four experiments.

Linkage map. A total of 96 polymorphic SSR markers, 247 polymorphic AFLP markers, and 346 polymorphic RFLP markers were scored in the DH population 307-230-2/307-406-1. Due to the allopolyploid nature of the B. napus genome a large proportion of duplicated loci are expected, which can distort the marker segregation and lead to mapping errors (5); these can be recognized because the marker segregation ratios deviate significantly from the expected 1:1 ratio in a $\mathrm{DH}$ population. In order to obtain the best possible map, we therefore performed a stringent selection for markers with correct or only moderately skewed segregation, and to facilitate the composite interval mapping of QTL we further removed mapped markers with less than $1 \mathrm{cM}$ intervals. Overall these measures resulted in the exclusion of $53.1 \%$ of the available SSR markers, $61.1 \%$ of the AFLP markers, and $52.3 \%$ of the RFLP markers from the mapping. The resulting genetic map comprised 165 RFLP, 94 AFLP, and 45 SSR markers on 19 linkage groups (LG) covering $1,739 \mathrm{cM}$, with an average marker distance of $5.7 \mathrm{cM}$ (Fig. 2). A total of 68 markers $(22 \%)$ with moderately skewed distribution $(P<0.01)$ were included in the map, but only when they did not

TABLE 1. Comparison of mean least significant differences in Verticillium longisporum disease index values in a population of 163 doubled haploid Brassica napus lines, and their parents 307-230-1 (susceptible) and 307-230-2 (resistant), after root-dip inoculation in four experiments performed in the greenhouse in autumn 1999

\begin{tabular}{lcccc}
\hline & \multicolumn{3}{c}{ Mean disease index } & \multirow{2}{*}{ LSD $^{\mathrm{v}}$} \\
\cline { 2 - 4 } Experiment & $\mathrm{DH}^{\mathrm{w}}$ lines & $307-230-1$ & $307-406-2$ & $(\alpha=0.05)$ \\
\hline Exp. 1: IM & $3.45^{\mathrm{a}}$ & $2.47^{\mathrm{c}}$ & $4.49^{\mathrm{e}}$ & 0.63 \\
Exp. 2: $\mathrm{IM}^{\mathrm{y}}$ & $3.50^{\mathrm{a}}$ & $2.77^{\mathrm{c}}$ & $4.69^{\mathrm{e}}$ & 0.64 \\
Exp. 3: $\mathrm{IM}^{\mathrm{y}}$ & $3.53^{\mathrm{a}}$ & $2.76^{\mathrm{c}}$ & $4.73^{\mathrm{e}}$ & 0.63 \\
Exp. 4: $\mathrm{SSI}^{\mathrm{z}}$ & $3.00^{\mathrm{b}}$ & $2.17^{\mathrm{d}}$ & $4.21^{\mathrm{f}}$ & 0.66 \\
LSD $^{\mathrm{d}}(\alpha=0.05)$ & 0.30 & 0.37 & 0.25 & \\
\hline
\end{tabular}

${ }^{v}$ The disease index for each doubled haploid line in each experiment was calculated from the symptom scores of 21 inoculated and 21 noninoculated plants per genotype, while the mean disease index of the parental lines was calculated from 10 repetitions of 21 inoculated and 21 noninoculated plants in each experiment. Different mean values that show nonsignificant differences are assigned the same superscript letter.

${ }^{\mathrm{w}}$ Doubled-haploid.

${ }^{x}$ Least significant difference.

${ }^{\mathrm{y}}$ Isolate mixture.

${ }^{\mathrm{z}}$ Single spore isolate. form noticeable clusters. Based on comparisons to other maps, all linkage groups could be associated to defined B. napus chromosomes based on the accepted N1-N19 nomenclature for B. napus (22). The size of the groups varied between 46.3 and $158.1 \mathrm{cM}$, while the number of molecular markers per group ranged from 3 to 35 . A maximum of seven SSR markers were identified for each linkage group.

QTL for V. longisporum resistance. Four chromosome regions were identified that contained significant QTL for resistance to $V$. longisporum (defined as significant LOD scores after multiple regression combined with significant phenotypic effects at a threshold of $\theta=0.02$ ) in at least two of the four experiments (Fig. 2 ). In all cases the resistance alleles at the detected QTL were derived from the resistant parent 307-230-2. The relative degree of differentiation in disease response within this mapping population was comparatively low, however together the four significant QTL explained a total of $45.7 \%$ (sum of partial $R^{2}$ ) of the phenotypic variance in the $V$. longisporum DI. To our knowledge this is the first report of QTL for resistance to $V$. longisporum in B. napus, hence these results represent an important first step in the investigation of the genetic basis of currently available resistance sources.

On N15, a QTL region with overlapping confidence intervals was detected in two of the three experiments with the isolate mixture, and also with the mean data for the three experiments with the isolate mixture (Table 3). A QTL whose confidence interval was adjacent to this region was detected in the other experiment with the isolate mixture. No significant QTL was detected at this position in the experiment with the single spore isolate, however the marker BRMS030 flanking the QTL had significant effects ( $t$ test, $P<0.05$ ) on the mean DI for the singlespore isolate. The power of QTL detection may have been lower in the experiment with the single-spore isolate, since the majority of the DH lines showed an intermediate resistance response; this could explain why no significant QTL was detected in this case.

On N14 another QTL region was found with overlapping QTL confidence intervals for one of the three experiments with the isolate mixture, the mean data over all environments with the isolate mixture, and for the experiment with the single-spore isolate. Although no significant QTL were detected at this position in the other two experiments with the isolate mixture, the flanking markers (DLM138b and CB10079) still both had significant effects on the mean DI in these replications ( $t$ test, $P<0.01$ ), suggesting that this region also contains one or more genes with a significant effect on the resistance reaction. The QTL for the experiment with the single-spore isolate was at a slightly different position to the QTL from the experiments with the isolate mixture, which might indicate a race-specific resistance gene that was no longer effective against the isolate mixture.

In the mean data from the three experiments with the isolate mixture, the two QTL on N14 and N15 accounted for 9.9 and $12.5 \%$ of the phenotypic variation (partial $R^{2}$ ), respectively. Because these two QTL had consistently significant effects on the

TABLE 2. Pearson's correlation coefficient for comparisons among Verticillium longisporum disease index values of 163 doubled haploid Brassica napus lines and their population parents tested by root-dip inoculation in three greenhouse experiments performed in autumn 1999 with an isolate mixture and in one experiment with a single-spore isolate ${ }^{\mathrm{x}}$

\begin{tabular}{lcccc}
\hline & & \multicolumn{3}{c}{ Exp. 1-3: } \\
Experiment & Exp. 2: IM & Exp. 3: IM & Mean & Exp. 4: SSI $^{\mathrm{z}}$ \\
\hline Exp. 1: IM & 0.64 & 0.68 & 0.87 & 0.64 \\
Exp. 2: IM & & 0.76 & 0.90 & 0.57 \\
Exp. 3: IM $^{\mathrm{y}}$ & & & 0.91 & 0.57 \\
Exp. 1-3: Mean & & & 0.66 \\
\hline
\end{tabular}

x All correlations were significant with $P<0.01$.

y Isolate mixture.

${ }^{\mathrm{z}}$ Single spore isolate. 
N1

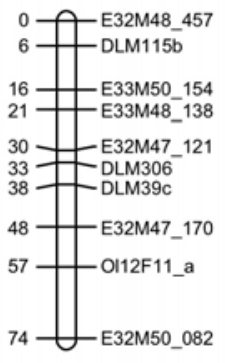

N2

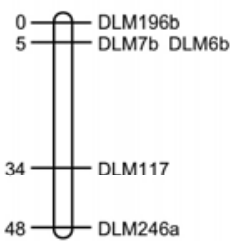

N3

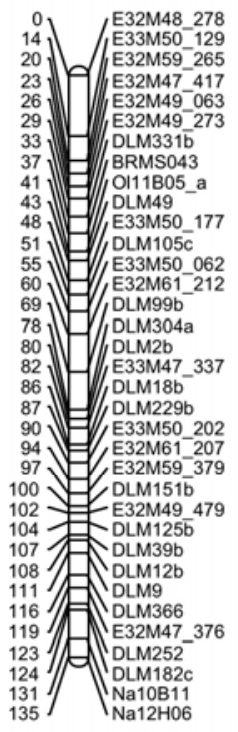

N4

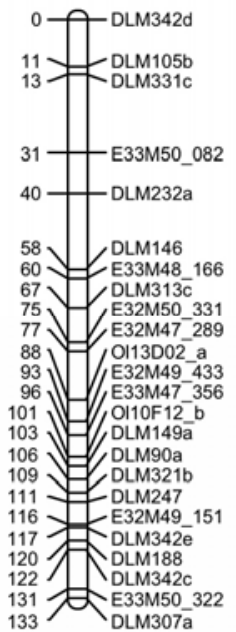

N5
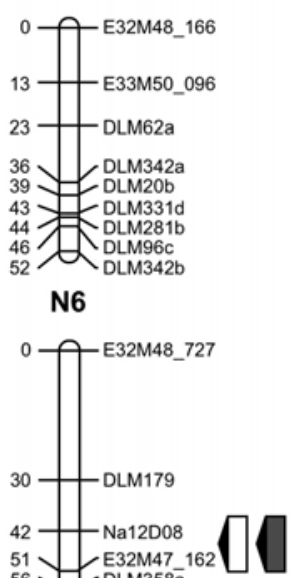

56 DLM358a

$63-D L M 274$

65 DLM174

69 DLM19a

${ }_{73}^{71}$ Na12C01_a

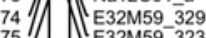

75 . E32M59-323

E32M50-470

105 E32M61-145

112 E32M62_061

N7

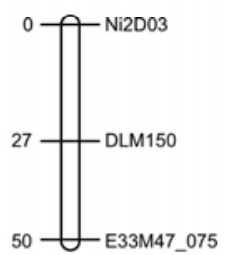

N8

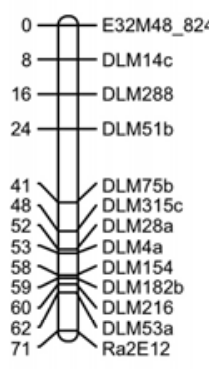

N9

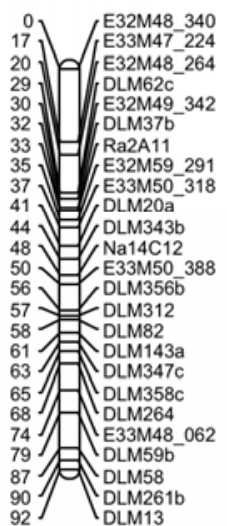

N10

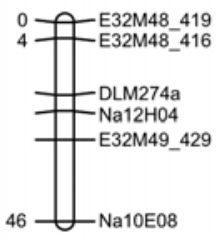

N11

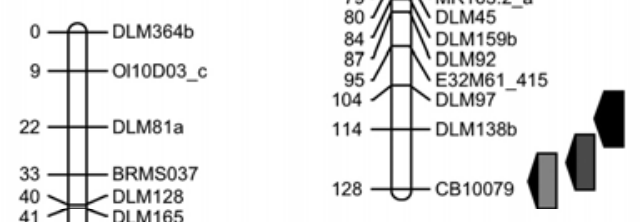

N12

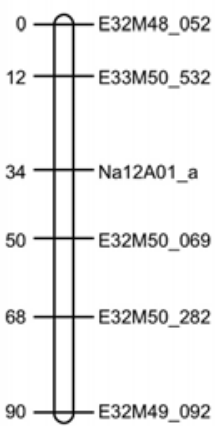

N13

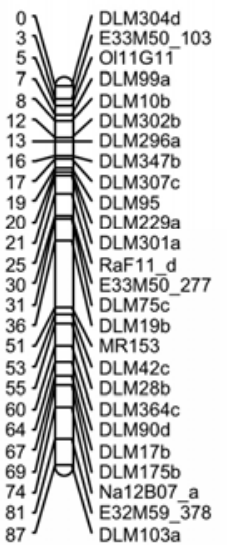

N14

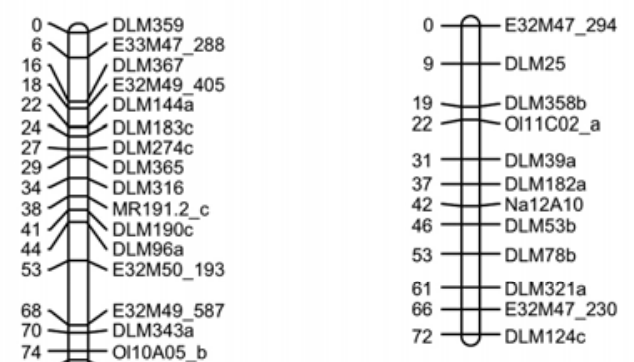

N15

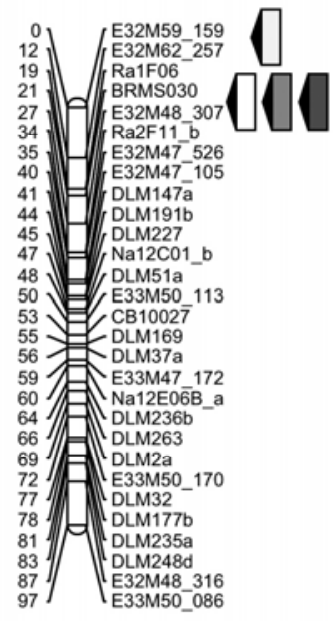

N16

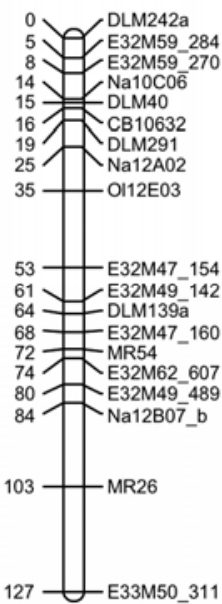

\section{N18}

28 $\iint_{\text {E32M61_070 }}^{\text {E32M61_066 }}$

33 Z DLM126a

${ }_{38}$. DLM323a

${ }_{42}^{38}$ DLM10c

${ }_{50}^{46}$ E $32 M 47_{\text {DLM14b }}^{244}$

57

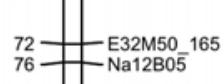

76 - $\mathrm{Na} 12 \mathrm{~B} 05$

$94-$ DLM129

$104-{ }^{N i 4 D 09}$

$109-$ DLM217a

${ }_{124}^{122}$ - $\begin{gathered}\text { DLM224 } \\ \text { DLM315e }\end{gathered}$

$131-$ DLM $324 b$

138 - E32M48_163

$146-$ E32M59_105

$158 \bigcup_{\text {DLM302a }}$
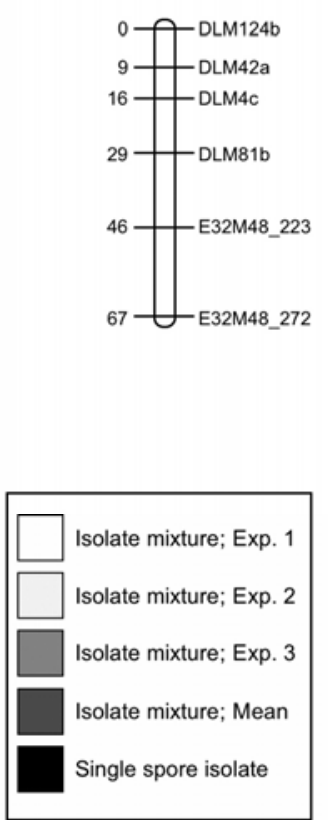

Fig. 2. Genetic linkage map (1,739 cM) for an oilseed rape mapping population (163 doubled haploid lines) derived from the population 307-230-2/307-406-1. The shaded boxes show quantitative trait loci for Verticillium longisporum disease index measured in the greenhouse in autumn 1999 after three root-dip inoculation experiments with a V. longisporum isolate mixture and one experiment with the single-spore isolate VD13. The map contains 165 restriction fragment length polymorphism, 94 amplified fragment length polymorphism and 45 simple sequence repeat markers. Genetic distances are given in centimorgans. 
resistance phenotype in multiple experiments with controlled but not identical inoculation and environmental conditions, we considered these to be environmentally stable QTL with potential for utilization in breeding. The B. napus chromosomes N14 and N15 are both derived from the Brassica C-genome (22), which is consistent with the origin of the resistance from a $B$. oleracea donor.

On the other hand, linkage groups N6 and N18 each contained one QTL region that showed significant phenotypic effects on resistance in a single experiment with the isolate mixture. Although the genetic effects of these QTL are apparently lower than for the QTL on N14 and N15, they nevertheless represent chromosomal regions of interest for future studies of $V$. longisporum resistance in oilseed rape. The QTL on N6, which accounted for $12.4 \%$ of the phenotypic variation (partial $R^{2}$ ) in the resistance reaction in the three experiments with the isolate mixture, is of particular interest because chromosome N6 in $B$. napus is derived from the A genome of $B$. rapa (21) rather than the $\mathrm{C}$-genome of the original $B$. oleracea resistance donor. This could be due to a homoeologous or nonhomoeologous translocation between $\mathrm{A}$ and $\mathrm{C}$ genome chromosomes, which are known to occur commonly in newly synthesised B. napus (29), or alternatively it could indicate a further resistance locus contributed by the $B$. rapa parent of the original RS B. napus resistance donor.

Expanding the genetic basis for resistance breeding in oilseed rape. The addition of new sources of resistance against $V$. longisporum to the germplasm of $B$. napus is vital for the sustainable production of oilseed rape in Europe, since there is only limited resistant germplasm currently available for breeding and Verticillium wilt is becoming increasingly important in some rapeseed production areas. Introgression of germplasm from $B$. rapa and $B$. oleracea via RS rapeseed is a breeding strategy that has been successfully used in the past to generate new genetic variation for resistance against important diseases of oilseed rape. For example, protoplast fusion has been successfully used to transfer resistance to clubroot disease (Plasmodiophora brassicae Wor.) from B. rapa to B. napus (7). Through advanced backcrossing a race-specific resistance was subsequently transferred from RS rapeseed to elite winter oilseed rape germplasm, and the winter oilseed rape cultivars Mendel and Tosca derived from this material were released in the early 2000s to specifically combat this disease in affected areas of Britain and Germany. Synthetic lines of $B$. napus carrying resistance to blackleg derived from $B$. rapa were generated via embryo culture (20). The resistance was subsequently introgressed into spring canola, resulting in the release of 'Surpass' in the late 1990s.

The introgression of novel resistance genes normally requires extensive backcrossing accompanied by effective resistance screening. However, selection for $V$. longisporum resistance in the field can be difficult due to a high environmental influence on the symptom development, and symptom scoring is further complicated by the frequent co-occurrence of blackleg disease caused by Leptosphaeria maculans (Desm) Ces. \& de Not. [anamorph Phoma lingam (Tode ex Fr.) Desm.] on plants showing V. longisporum symptoms (I. Happstadius, personal observation, 2007). As shown in Table 4, the combination of $V$. longisporum resistance alleles from different QTL in the present study was associated with increased resistance in the DH lines. This underlines the potential for a marker-assisted pyramiding strategy to assist the effective transfer of polygenic $V$. longisporum resistance from RS rapeseed donors into elite breeding lines.

TABLE 4. Numbers and mean Verticillium longisporum disease index values of doubled haploid Brassica napus lines containing zero, one, two, three, or four resistance alleles at the four quantitative trait loci (QTL) on B. napus chromosomes N6, N14, N15, and N18, compared with the disease index values of the susceptible 307-230-1 and the resistant 307-230-2v

\begin{tabular}{lccc}
\hline Genotypes & $\begin{array}{c}\text { Number of } \\
\text { resistance alleles } \\
\text { present }\end{array}$ & $\begin{array}{c}\text { Number of } \\
\text { DH }^{\mathrm{w}} \text { lines }^{\mathrm{x}}\end{array}$ & $\begin{array}{c}\text { Mean DI } \\
\text { value }\end{array}$ \\
\hline (307-230-2 / 307-230-1) & 0 & 1 & 4.62 \\
& 1 & 58 & 3.69 \\
& 2 & 47 & 3.39 \\
$307-230-1$ & 3 & 15 & 2.74 \\
$307-230-2$ & 4 & 4 & 2.36 \\
& 0 & - & $4.64^{\mathrm{z}}$ \\
\hline
\end{tabular}

${ }^{v}$ The disease index values were calculated from the symptom scores of 21 inoculated and 21 noninoculated plants per genotype. Data is from three root-dip inoculation experiments with an isolate mixture. Thirty-eight double haploid lines contained alleles from both parents at the QTL-flanking markers and were not counted.

${ }^{w}$ Doubled haploid.

${ }^{\mathrm{x}}$ Lines were counted as containing a resistance allele when the alleles for both markers flanking the QTL were derived from 307-230-2. The resistance allele was counted as absent when the alleles for both markers flanking a QTL were derived from 307-406-1.

y Disease index.

${ }^{\mathrm{z}}$ Mean DI values for the parental lines were calculated from 10 repetitions of 21 inoculated and 21 noninoculated plants in each experiment.

TABLE 3. Details of significant quantitative trait loci for resistance against Verticillium longisporum estimated from mean disease index data in a population of 163 doubled-haploid lines from the Brassica napus population 307-230-2/307-406-1

\begin{tabular}{|c|c|c|c|c|c|c|c|}
\hline Experiment $^{t}$ & $\begin{array}{l}\text { Linkage } \\
\text { group }\end{array}$ & $\begin{array}{l}\text { Peak position } \\
(\mathrm{cM})^{\mathrm{u}}\end{array}$ & $\begin{array}{c}\mathrm{LOD}^{\mathrm{v}} \text { at } \\
\mathrm{QTL}^{\mathrm{w}} \text { position }\end{array}$ & $\begin{array}{c}\text { Confidence } \\
\text { interval }(\mathrm{cM})^{\mathrm{u}}\end{array}$ & Flanking markers ${ }^{\mathrm{x}}$ & $\begin{array}{c}\text { Partial } R^{2} \\
(\%)\end{array}$ & $\begin{array}{l}\text { Additive } \\
\text { effects }\end{array}$ \\
\hline \multirow[t]{2}{*}{ Exp. 1: IM ${ }^{y}$} & N6 & 50 & 6.32 & $46-56$ & Na12D08 (41.8) - E32M47_162 (51.2) & 9.5 & 0.29 \\
\hline & N15 & 22 & 4.98 & $18-24$ & BRMS030 (21.4) - E32M48_307 (26.6) & 6.6 & 0.24 \\
\hline \multirow[t]{3}{*}{ Exp. 3: IM ${ }^{y}$} & N14 & 128 & 6.64 & $120-128$ & DLM138b (114.3) - CB10079 (128.5) & 8.6 & 0.24 \\
\hline & N15 & 22 & 6.68 & $18-24$ & BRMS030 (21.4) - E32M48_307 (26.6) & 9.7 & 0.27 \\
\hline & N18 & 130 & 3.30 & $126-136$ & DLM315e (124.3) - DLM324b (131.5) & 7.3 & 0.23 \\
\hline \multirow[t]{2}{*}{ Exp. 1-3: Mean } & N6 & 50 & 4.87 & $46-56$ & Na12D08 (41.8) - E32M47_162 (51.2) & 12.4 & 0.27 \\
\hline & N18 & 130 & 4.20 & $124-134$ & DLM315e (124.3) - DLM324b (131.5) & 10.9 & 0.24 \\
\hline Exp. 4: $\mathrm{SSI}^{\mathrm{z}}$ & N14 & 112 & 6.50 & $104-122$ & DLM97 (102.5) - DLM138b (112.9) & 7.1 & 0.25 \\
\hline
\end{tabular}

${ }^{t}$ The population was evaluated in four experiments representing different environments. Disease index of each genotype in each experiment was calculated by comparing disease symptoms of 21 plants inoculated by a root-dip procedure with 21 noninoculated plants. Only resistance loci with significant logarithm of odds scores after regression analysis in combination with significant phenotypic effects in more than one experiment are shown.

u Centimorgan.

${ }^{\mathrm{v}}$ Logarithm of odds.

${ }^{\mathrm{w}}$ Quantitative trait loci.

${ }^{x}$ In all cases, the marker alleles associated with increased resistance were derived from the resistant parent 307-230-2.

${ }^{\mathrm{y}}$ Isolate mixture.

${ }^{\mathrm{z}}$ Single spore isolate. 


\section{ACKNOWLEDGMENTS}

This work was partially supported by the Society for Promotion of Private German Plant Breeding (GFP) and the Agency for Renewable Resources (FNR) of the Federal Ministry for Nutrition, Agriculture and Consumer Protection (BMELV). We thank D. Baetzel for excellent technical assistance and four anonymous reviewers for constructive comments on an earlier version of this manuscript.

\section{LITERATURE CITED}

1. Badani, A. G., Snowdon, R. J., Baetzel, R., Lipsa, F. D., Wittkop, B., Horn, R., De Haro, A., Font, R., Lühs, W., and Friedt, W. 2006. Colocalization of a partially dominant gene for yellow seed color with a major QTL influencing acid detergent fiber (ADF) content in different crosses of oilseed rape (Brassica napus). Genome 49:1499-1509.

2. Basunanda, P., Spiller, T. H., Hasan, M., Gehringer, A., Schondelmaier, J., Lühs, W., Friedt, W., and Snowdon, R. J. 2007. Marker-assisted increase of genetic diversity in a double-low seed quality winter oilseed rape genetic background. Plant Breed. 126:581-587.

3. Berg, E. S., and Olaisen, B. 1994. Hybrid PCR sequencing - sequencing of PCR products using a universal primer. Biotechniques 17:896.

4. Cheung, W. Y., Champagne, G., Hubert, N., and Landry, B. S. 1997. Comparison of the genetic maps of Brassica napus and Brassica oleracea. Theor. Appl. Genet. 94:569-582.

5. Cloutier, S., Cappadocia, M., and Landry, B. S. 1997. Analysis of RFLP mapping inaccuracy in Brassica napus L. Theor. Appl. Genet. 95:83-91.

6. Debode, J., Declercq, B., and Höfte, M. 2005. Identification of cauliflower cultivars that differ in susceptibility to Verticillium longisporum using different inoculation methods. J. Phytopathol. 153:257-263.

7. Diederichsen, E., and Sacristan, M. D. 1996. Disease response of resynthesized Brassica napus L. lines carrying different contributions of resistance to Plasmodiophora brassicae Wor. Plant Breed. 115:5-10.

8. Doyle, J. J., and Doyle, J. L. 1990. Isolation of plant DNA from fresh tissue. Focus 12:13-15.

9. Dunker, S., Keunecke, H., and von Tiedemann, A. 2006. Verticillium longisporum in winter oilseed rape - Impact on plant development and yield. Integrated Control in Oilseed Crops 29:365-374.

10. Eynck, C., Koopmann, B., Grunewaldt-Stoecker, G., Karlovsky, P., and von Tiedemann, A. 2007. Differential interactions of Verticillium longisporum and $V$. dahliae with Brassica napus detected with molecular and histological techniques. Eur. J. Plant Pathol. 118:259-274.

11. Happstadius, I., Ljungberg, A., Kristiansson, B., and Dixelius, C. 2003. Identification of Brassica oleracea germplasm with improved resistance to Verticillium wilt. Plant Breed. 122:30-34.

12. Hasan, M., Seyis, F., Badani, A. G., Pons-Kuhnemann, J., Lühs, W., Friedt, W., and Snowdon, R. J. 2006. Surveying genetic diversity in the Brassica napus gene pool using SSR markers. Genet. Res. Crop Evol. 53:793-802.

13. Johansson, A., Staal, J., and Dixelius, C. 2006. Early responses in the Arabidopsis-Verticillium longisporum pathosystem are dependent on NDR1, JA- and ET-associated signals via cytosolic NPR1 and RFO1. Mol. Plant-Microbe Interact. 19:958-969.

14. Karapapa, V. K., Bainbridge, B. W., and Heale, J. B. 1997. Morphological and molecular characterization of Verticillium longisporum comb. nov., pathogenic to oilseed rape. Mycol. Res. 101:1281-1294.

15. Koike, S. T., Subbarao, K. V., Davis, R. M., Gordon, T. R., and Hubbard, J. C. 1994. Verticillium wilt of cauliflower in California. Plant Dis. 78:1116-1121.

16. Kosambi, D. D. 1944. The estimation of map distances from recombination values. Ann. Eugen. 12:172-175.

17. Landry, B. S., Hubert, N., Etoh, T., Harrada, J. J., and Lincoln, S. E. 1991. A genetic map for Brassica napus based on restriction fragment length polymorphisms detected with expressed DNA sequences. Genome 34:543-552.

18. Lombard, V., Baril, C. P., Dubreuil, P., Blouet, F., and Zhang, D. 2000. Genetic relationships and fingerprinting of rapeseed cultivars by AFLP: Consequences for varietal registration. Crop Sci. 40:1417-1425.

19. Lowe, A. J., Moule, C. L., Trick, M., and Edwards, K. J. 2004. Efficient large-scale development of microsatellites for marker and mapping applications in Brassica crop species. Theor. Appl. Genet. 108:11031112.

20. Mithen, R. F., and Magrath, R. 1992. Glucosinolates and resistance to Leptosphaeria maculans in wild and cultivated Brassica species. Plant Breed. 108:60-68.

21. Parkin, I. A. P., Sharpe, A. G., Keith, D. J., and Lydiate, D. J. 1995. Identification of the $\mathrm{A}$ and $\mathrm{C}$ genomes of amphidiploid Brassica napus (oilseed rape). Genome 38:1122-1131.

22. Parkin, I. A. P., Sigrun, M. G., Sharpe, A. G., Lukens, L., Trick, M., Osborn, T. C., and Lydiate, D. J. 2005. Segmental structure of the Brassica napus genome based on comparative analysis with Arabidopsis thaliana. Genetics 171:765-781.

23. Piquemal, J., Cinquin, E., Couton, F., Rondeau, F., Seignoret, E., Doucet, I., Perret, D., Villeger, M. J., Vincourt, P., and Blanchard, P. 2005. Construction of an oilseed rape (Brassica napus L.) genetic map with SSR markers. Theor. Appl. Genet. 111:1514-1523.

24. Rygulla, W., Seyis, F., Eynck, C., Friedt, W., Lühs, W., Snowdon, R., and von Tiedemann, A. 2007. Generation of zero erucic acid resynthesised rapeseed lines with resistance to Verticillium longisporum. Plant Breed. 126:596-602.

25. Rygulla, W., Snowdon, R. J., Eynck, C., von Tiedemann, A., Lühs, W., and Friedt, W. 2007. Broadening the genetic basis of Verticillium longisporum resistance in Brassica napus by interspecific hybridization. Phytopathology 97:1391-1396.

26. Snowdon, R. J., Lühs, W., and Friedt, W. 2006. Oilseed rape. Genome Mapping and Molecular Breeding, Vol. 2. Pages 55-114 in: Oilseeds. C. Kole, ed. Springer Verlag, Heidelberg, New York, Berlin, Toyko.

27. Steventon, L. A., Fahleson, J., Hu, Q., and Dixelius, C. 2002. Identification of the causal agent of Verticillium wilt of winter oilseed rape in Sweden, V. longisporum. Mycol. Res. 106:570-578.

28. Steventon, L. A., Happstadius, I., Okori, P., and Dixelius, C. 2002. Development of a rapid technique for the evaluation of the response of Brassica napus to Verticillium wilt. Plant Dis. 86:854-858.

29. Udall, J. A., Quijada, P. A., and Osborn, T. C. 2005. Detection of chromosomal rearrangements derived from homoeologous recombination in four mapping populations of Brassica napus L. Genetics 169:967-979.

30. Utz, H. F., and Melchinger, A. E. 1996. PLABQTL: A program for composite interval mapping of QTL. J. Quant. Trait Loci 2:1.

31. Van Ooijen, J. W., and Voorrips, R. E. 2001. JoinMap 3.0, Software for the calculation of genetic linkage maps. Plant Research International, Wageningen, The Netherlands.

32. Veronese, P., Narasimhan, M. L., Stevenson, R. L., Zhu, J. K., Weller, S. C., Subbarao, K. V., and Bressan, R. A. 2003. Identification of a locus controlling Verticillium disease symptom response in Arabidopsis thaliana. Plant J. 35:574-587.

33. Weber, S., Ünker, F., and Friedt, W. 2005. Production of Brassica napus (L.) doubled haploids by microspore in vitro colchicine treatment and determination of ploidy by flow cytometry. Plant Breed. 124:511-513.

34. Zeise, K., and von Tiedemann, A. 2002. Host specialization among vegetative compatibility groups of Verticillium dahliae in relation to Verticillium longisporum. J. Phytopathol. 150:112-119.

35. Zhou, L., Hu, Q., Johansson, A., and Dixelius, C. 2006 Verticillium longisporum and V. dahliae: Infection and disease in Brassica napus. Plant Pathol. 55:137-144.

36. Zhou, W. J., Tang, G. X., and Hagberg, P. 2002. Efficient production of doubled haploid plants by immediate colchicine treatment of isolated microspores in winter Brassica napus. Plant Growth Regul. 37:185-192. 